$\underline{\text { Preprint typeset in JHEP style. - HYPER VERSION }}$

hep-ph/0009001

\title{
A Light Bottom Squark in the MSSM
}

\author{
Athanasios Dedes and Herbi K. Dreiner \\ Rutherford Appleton Laboratory \\ Chilton, Didcot, OX11 OQX, UK \\ E-mail: a.dedes@rl.ac.uk, h.k.dreiner@rl.ac.uk
}

\begin{abstract}
We study the compatibility of a light bottom squark $M_{\tilde{b}}<\mathcal{O}(10 \mathrm{GeV})$ in the unconstrained MSSM. We consider the one-loop radiative corrections which are large for a heavy gluino $(>\mathcal{O}(150 \mathrm{GeV}))$. We then consider the renormalization group flow up to the Grand Unified scale. For most regions of the parameter space with a light sbottom we find colour and charge breaking minima. Only a small window in gluino mass and $\tan \beta$ is consistent with all bounds. This is alleviated by a light gluino, which is however only marginally experimentally allowed.
\end{abstract}

Keywords: Beyond Standard Model, Supersymmetric Standard Model. 


\section{Contents}

1. Introduction 1

2. Parameters and Constraints 2

3. Sbottom Pole Mass 4

4. Renormalization Group 6

$4.1 \quad$ Universal Scalar Masses at $M_{X}$

4.2 Non-Universal Scalar Masses at $M_{X}$

5. Light Gluino 10

6. Conclusions 11

\section{Introduction}

The experimental bound from LEP on the lightest supersymmetric particle (LSP), assuming it is a neutralino, is given by [1]

$$
M_{\tilde{\chi}_{1}^{0}}>40.9 \mathrm{GeV} ; \quad(\mathrm{OPAL}),
$$

and similar numbers from DELPHI $(36.7 \mathrm{GeV})$ [2], L3 $(38.2 \mathrm{GeV})$ [3] and ALEPH $(37.5 \mathrm{GeV})$ [4]. This bound assumes the supersymmetric grand unified relation between the gaugino masses at the weak scale: $M_{1}=(5 / 3) \tan ^{2} \theta_{W} M_{2}$ (where $\theta_{W}$ is the electroweak mixing angle) and employs the chargino search. In a recent paper [5] it was shown that if you drop this theoretical assumption a LSP neutralino even as light as $34 \mathrm{MeV}$ is consistent with all experiments. ${ }^{1}$

It is the purpose of this letter to do a similar study for light bottom squarks. Light top squarks have been extensively studied elsewhere [7]. For large values of $\tan \beta$ (the ratio of the vacuum expectation values of the two neutral CP-even Higgs bosons in the MSSM) it can be natural to have light bottom squarks as well, as we discuss in more detail below. Both the D0 and CDF experiments have performed direct searches for the lightest bottom squark [8, 9] obtaining

$$
\begin{array}{ll}
m_{\tilde{b}}>115 \mathrm{GeV}, & (\mathrm{D} 0) \\
m_{\tilde{b}}>146 \mathrm{GeV}, & (\mathrm{CDF}) .
\end{array}
$$

\footnotetext{
${ }^{1}$ For a discussion of astrophysical bounds see $[5,6]$.
} 
We have given the maximum bound which is obtained for a vanishing neutralino LSP mass. In general the bound depends on the LSP mass and becomes less sensitive as the mass difference between the LSP and the squark is decreased. For smaller mass differences and also for smaller bottom squark masses the LEP searches [10, 11, 12, 13, 14 are more sensitive. However, even in this case there remains a gap at very small mass differences to the LSP which becomes more pronounced for very small squark masses $m_{\tilde{b}}<\mathcal{O}(10 \mathrm{GeV})$ [14]. At such low masses the decay $\tilde{b} \rightarrow b \tilde{\chi}_{1}^{0}$ is kinematically suppressed by the final state quark even for vanishing neutralino mass. A dedicated search for the top squark with a small mass difference $(\Delta M)$ to the LSP has been performed [15] reaching as low as $\Delta M=1.6 \mathrm{GeV}$; the threshold for the decay $\tilde{t} \rightarrow \tilde{\chi}_{1}^{0} c$. We are not aware of such a search for light bottom squarks.

Light squarks can directly contribute to the hadronic cross section at the $Z^{0}$ peak. As we will discuss below, the doublet and singlet squarks mix and for specific mixing parameters the coupling to the $Z^{0}$ can even vanish. Thus this constraint restricts the range of sbottom mixing but can not exclude a light sbottom. This constraint turns out to be very mild since the light sbottom is dominantly an $S U(2)_{L}$ singlet.

Very light bottom squarks have recently been investigated in Refs. [16, 17]. In [16] a possible influence on the parameter $R(s)=\sigma\left(e^{+} e^{-} \rightarrow\right.$ hadrons $) / \sigma\left(e^{+} e^{-} \rightarrow\right.$ $\mu^{+} \mu^{-}$) was studied. For a $b$-squark the asymptotic contribution is only $1 / 12$. This is $1 / 4$ that of a b-quark due to the missing spin degeneracy and is below the experimental sensitivity [18]. In [17] the effect of a light b-squark on the electroweak precision data and on the MSSM Higgs sector was investigated. It was found to be consistent with the precision data provided the scalar top quark is not too heavy. The upper bound on the lightest CP-even Higgs boson in the MSSM is slightly lowered.

In the following we discuss the theoretical implications of a very light bottom squark. We focus on the embedding into the MSSM. We first study the pole mass of the bottom squark at one-loop, including in particular radiative corrections from the gluino which are large, and also corrections from top and bottom Yukawa couplings to the Higgs boson masses. We then study the renormalization group flow of the right-handed bottom squark mass squared for both universal and non-universal scalar fields at the GUT scale. We consider the constraints from colour and charge breaking minima (CCB). The constraints are relaxed by a light gluino. We finish with a brief discussion of bounds on a light gluino, before we conclude.

\section{Parameters and Constraints}

In the MSSM there are two bottom squarks. The $S U(2)_{L}$ current eigenstates are denoted $\tilde{b}_{L}, \tilde{b}_{R}$, where $\tilde{b}_{L}$ is a doublet squark and $\tilde{b}_{R}$ is a singlet squark. The corresponding states for the top squark are $\tilde{t}_{L}, \tilde{t}_{R}$. The mass matrix of these squarks in the current eigenstate basis is given for example in [19] and the one-loop radiative corrections are given in [20]. 
The mass eigenstates depend on the following parameters in the standard MSSM notation [21]: $M_{\tilde{Q}, \tilde{D}, \tilde{U}}^{2}$, the doublet and singlet soft-breaking squark masses, respectively; $M_{W, Z}$, the gauge boson masses; $\tan \beta$, the ratio of the vacuum expectation values of the two neutral CP-even Higgs fields; $A_{b}, A_{t}$, the tri-linear soft breaking terms; $\mu$, the Higgs mixing parameter; and $M_{\tilde{g}}$, the gluino mass. In the following $m_{b}$ and $m_{t}$ denote the bottom and top quark mass, respectively. We shall denote the lighter bottom squark $\tilde{b}_{2}$ and the heavier one $\tilde{b}_{1}$ in accordance with [20]. The scalar bottom mixing angle we denote $\theta_{\tilde{b}}$. All the above parameters are considered to be $\overline{D R}$-running parameters.

Besides the direct searches we have discussed in the introduction a light b-squark would also contribute to the hadronic cross section at the $Z^{0}$ peak, $\sigma_{\text {had }}^{0}$. The experimental bound for any contribution beyond the SM is $[18,22]^{2}$

$$
\Delta \sigma_{\text {had }}^{0}\left(Z^{0}\right)<0.142 n b, \quad(2 \sigma) .
$$

At tree-level this requires the sbottom mixing angle to lie in the range (for $\sin \theta_{W}^{2}=$ 0.2315 and $\left.N_{c}=3\right)$

$$
\left|\sin \theta_{\tilde{b}}\right|<0.535 .
$$

At $2 \sigma$, zero mixing is consistent with the data. In our analysis we have included the one-loop contribution to $\sigma_{\text {had }}^{0}$ from the scalar bottom. We have only plotted points which are consistent with the bound (2.2). It turns out that this constraint has no effect on Figs. 1 1 . For $\tilde{b}_{2}$ satisfying (2.2) the heavier bottom squark, $\tilde{b}_{1}$, couples unsuppressed to both the photon and the $Z^{0}$. In order to avoid experimental bounds from LEP1 and LEP2 we must therefore require

$$
m_{\tilde{b}_{1}} \gtrsim 200 \mathrm{GeV} \text {. }
$$

In our scans below, we shall employ this bound, as well.

A light sbottom contributes to the running of the strong coupling $\alpha_{s}$ between $m_{\tau}$ and $M_{Z^{0}}$. In order to see whether this is consistent with the data one must include the light sbottom both in the determination of $\alpha_{s}$ in a given experiment and also in the beta function. This is beyond the scope of this letter. However, it has been performed for a light gluino [23, 24]. The most recent study [24] with the smallest experimental error in $\alpha_{s}$ was able to exclude a light gluino at the $70 \%$ C.L.. The contribution to the beta function at one-loop of a singlet sbottom is $1 / 12$ that of a gluino. We thus expect the effect to be significantly smaller and beyond present experimental sensitivity.

\footnotetext{
${ }^{2}$ We note that the Standard Model prediction of $\sigma_{\text {had }}^{0}$ is currently $1.7 \sigma$ below the measured value. In principle this could be exactly compensated by the light bottom squark [17]. In the following we choose to focus only on the experimental upper bound on a new contribution.
} 

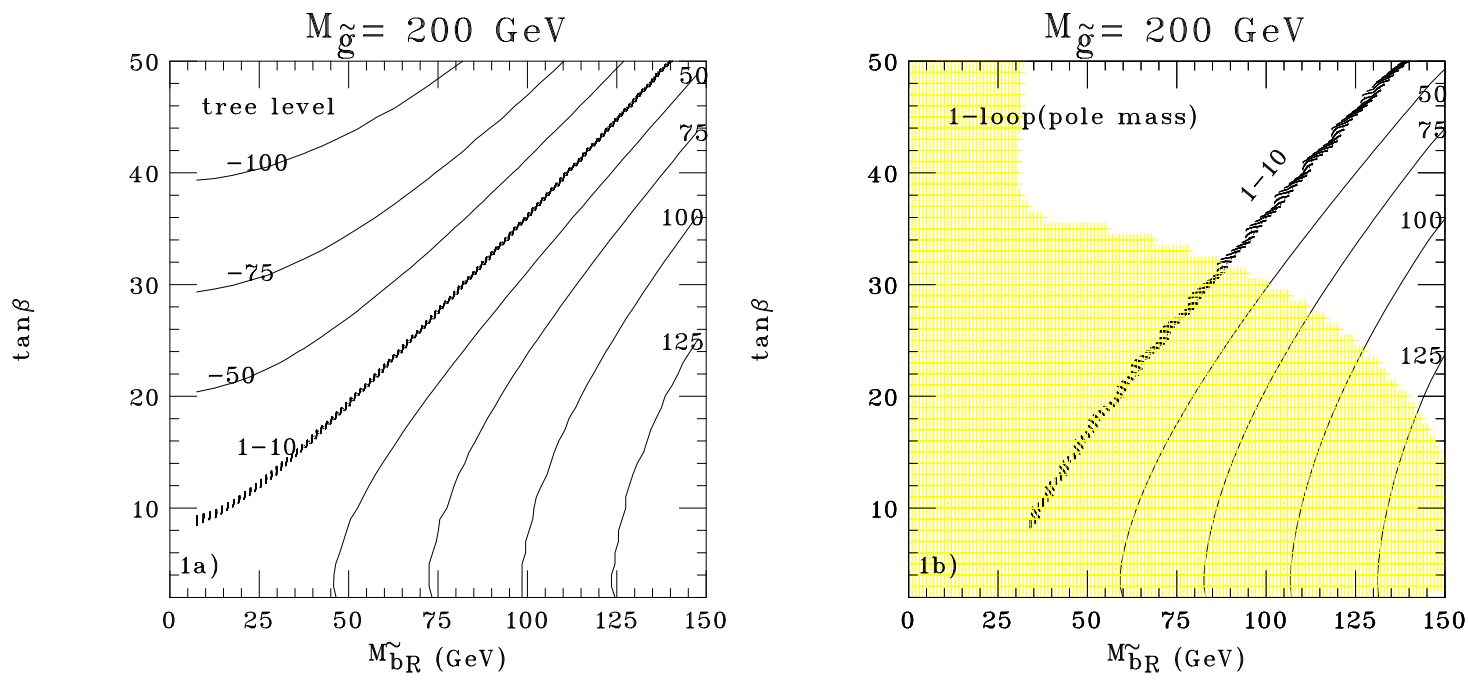

Figure 1: a) Contour plot of the tree level light sbottom mass $m_{\tilde{b}_{2}}$ as a function of the right handed singlet soft SUSY breaking mass $M_{\tilde{b}_{R}}$ and $\tan \beta$. The tree level mass-squared $\operatorname{sign}\left(m_{\tilde{b}_{2}}^{2}\right)\left|m_{\tilde{b}_{2}}^{2}\right|^{1 / 2}$ is getting negative for $\tan \beta \geq 10$ and for various values of $M_{\tilde{b}_{R}}$ indicated in the figure. The small dashed band indicates values of the light sbottom in the region $1-10 \mathrm{GeV}$. The soft trilinear coupling $A_{b}$ has been set to zero in this plot. b) As in Fig.1a but for the physical 1-loop mass. Sbottom masses of 50,75, 100, $125 \mathrm{GeV}$ are indicated for comparison. In the large shaded region, the running $\overline{D R}$ mass squared $\left(M_{\tilde{b}_{R}}^{2}\right)$ is getting negative at a scale which lies below the GUT scale.

\section{Sbottom Pole Mass}

We now investigate the effect of radiative corrections on the sbottom pole mass. $m_{\tilde{b}_{2}}$ depends at tree-level on the parameters $M_{\tilde{Q}}, M_{\tilde{D}}, A_{b}, \mu$ and $\tan \beta$. At oneloop [20], there is a further dependence on the stop mass parameters, $M_{\tilde{g}}$, and $M_{A}{ }^{3}$ The dependence on the stop sector and the Higgs sector parameters is weak and we fix them to $A_{t}=300 \mathrm{GeV}, M_{\tilde{t}_{R}}=300 \mathrm{GeV}$ and $M_{A}=400 \mathrm{GeV}$. We also fix the following SM parameters at the $Z^{0}$ scale to: $m_{t}($ pole $)=175 \mathrm{GeV}, \sin ^{2} \theta_{w}=$ $0.2315, m_{b}=2.9 \mathrm{GeV}$, and $\alpha_{s}=0.12$. As we discuss now, the dependence on $M_{\tilde{b}_{R}}$, $A_{b}$ and on $M_{\tilde{g}}$ is strong.

The first case we examine is that of a heavy gluino of $200 \mathrm{GeV}$, just above the current experimental bound of $180 \mathrm{GeV}$ [25]. For now, we fix the remaining input parameters: $M_{\tilde{b}_{L}}=250 \mathrm{GeV}, A_{b}=0 \mathrm{GeV}, \mu=250 \mathrm{GeV}$, at the $Z^{0}$ scale. In Fig. 17a,b we present contour plots of the lightest bottom squark mass, $m_{\tilde{b}_{2}}$ in the $\left(M_{\tilde{b}_{R}}\right.$-tan $\left.\beta\right)$ plane. We display both tree level and physical 1-loop pole masses. The narrow

\footnotetext{
${ }^{3}$ We do not include chargino-neutralino corrections since they are small [20]. Also, the light Higgs mass has been set to $M_{Z}$. Variation of the Higgs mass to its upper limit affects very weakly the light sbottom mass.
} 

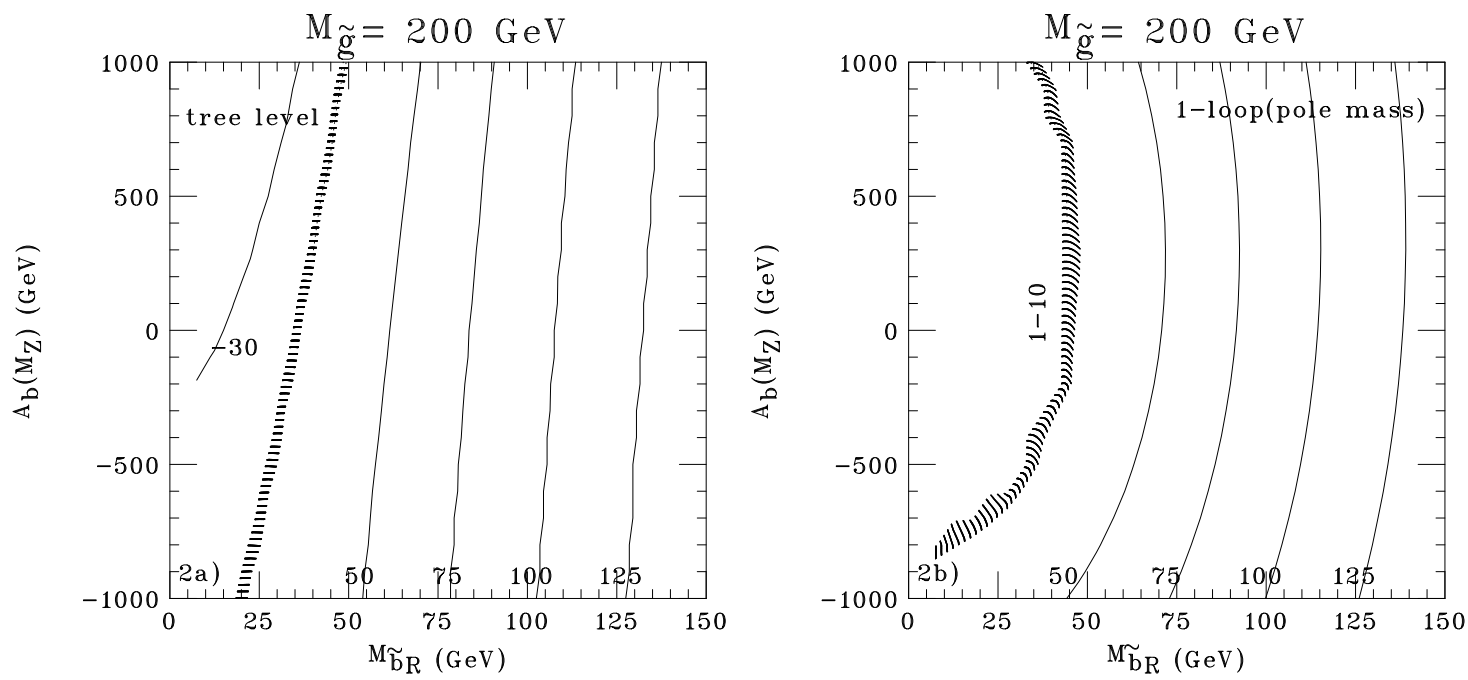

Figure 2: a) Contour plot of the tree level light sbottom mass as a function of the right handed singlet soft SUSY breaking mass $M_{\tilde{b}_{R}}$ and the trilinear coupling $A_{b}\left(M_{Z}\right)$. The value of $\tan \beta$ is fixed to 15 . 2b) The same for the physical 1-loop light sbottom pole mass. The light sbottom mass contours, $1-10 \mathrm{GeV}$ of this figure is completely within the shaded region of Fig.1b.

shaded strip corresponds to masses in the range $1-10 \mathrm{GeV}$. The area to the left of this narrow strip in Fig. 1b is excluded since the scalar bottom pole mass squared turns out to be negative. The effect of the radiative corrections is significant for $\tan \beta \lesssim 15$. One can see up to a $40 \mathrm{GeV}$ difference between the tree level and the 1-loop physical mass. They tend to push a fixed sbottom mass to larger values of $M_{\tilde{b}_{R}}$. The solution region for a light sbottom is quite narrow and somewhat finetuned. A variation of $1 \mathrm{GeV}$ of $M_{\tilde{b}_{R}}$ results in a variation of more than $5 \mathrm{GeV}$ in the light sbottom mass in the $\mathcal{O}(<10 \mathrm{GeV})$ region. Thus when determining the supersymmetric parameters for a light bottom squark the radiative corrections need to be taken into account.

With the above input values we obtain for the other (physical 1-loop) masses : $m_{\tilde{b}_{1}}=255-300 \mathrm{GeV}, m_{\tilde{t}_{1}}=191-228 \mathrm{GeV}, m_{\tilde{t}_{2}}=394-416 \mathrm{GeV}$. All these masses satisfy the current experimental bounds.

As we have already mentioned above, another parameter which plays a crucial role in determining the mass of the bottom squark is the trilinear coupling $A_{b}\left(M_{Z}\right)$. This parameter enters in both tree level and 1-loop sbottom mass corrections and the effect of its variation is presented in Fig. 2. The input parameter $\tan \beta$ is fixed to $\tan \beta=15$. As we can see, the one loop radiative corrections shift the mass contours by at most tens of $G e V$. Large values of $A_{b}\left(M_{Z}\right)$ typically give smaller sbottom masses. The other physical masses $\left(m_{\tilde{b}_{1}}, m_{\tilde{t}_{1}}, m_{\tilde{t}_{2}}\right)$ vary inside the region we mentioned in the previous paragraph. 

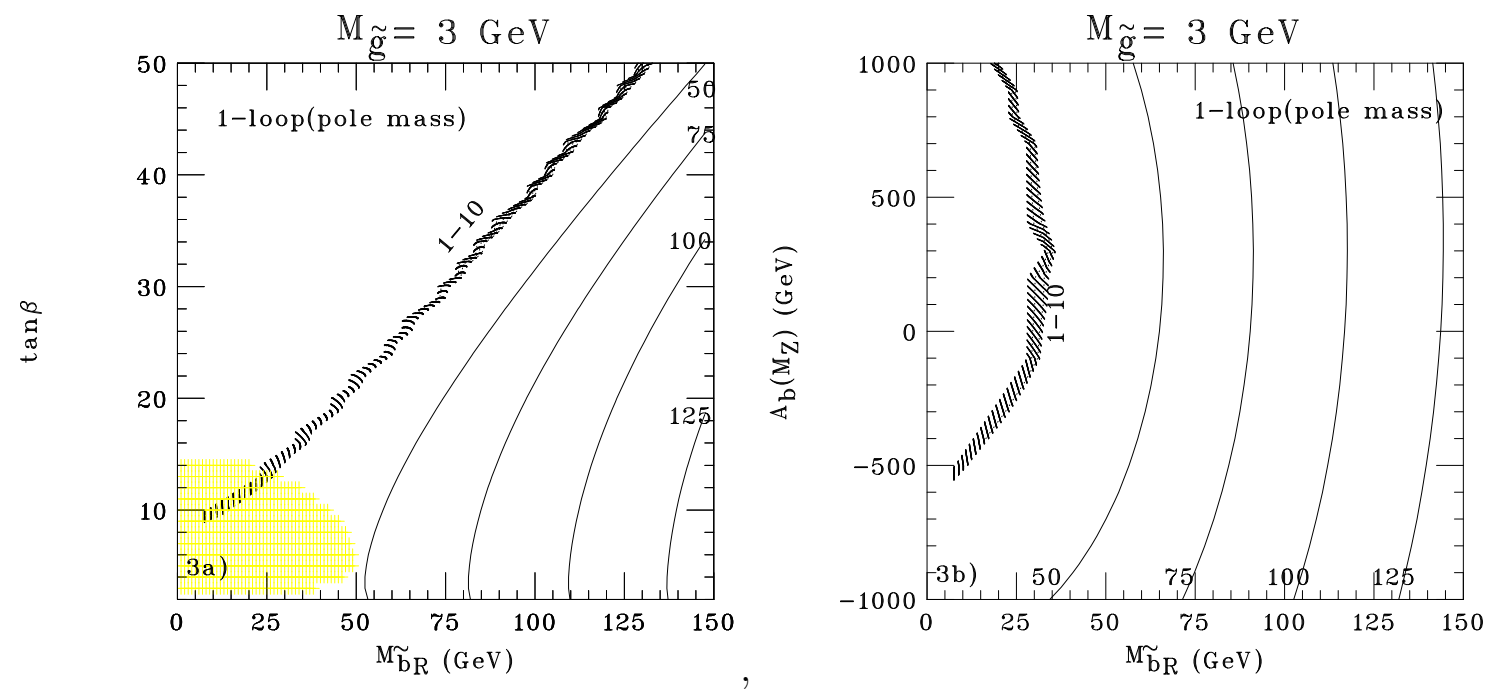

Figure 3: a) The same as in Fig.1b 3b) and in Fig.2b but for a light gluino of mass, $M_{\tilde{g}}=3 \mathrm{GeV}$. The tree level results are those given in Fig.1a and Fig.2a .

Further important radiative correction to the bottom squark masses are those arising from the loops involving gluinos [20]. This is obvious when one compares the Figs. 1, 20 where the gluino mass is taken to be $200 \mathrm{GeV}$ with Fig. 3 where its mass is set to be $3 \mathrm{GeV}$. We see that in the $\left(M_{\tilde{b}_{R}}-\tan \beta\right)$ plane there is almost no effect from radiative corrections for a light gluino compared to the tree-level result presented in Fig.11a. In the $\left(M_{\tilde{b}_{R}}-A_{b}\right)$ plane the effect is much less dramatic for a light gluino, although there is still a qualitative difference in the $A_{b}$ dependence compared to the tree-level result.

In summary, a light bottom squark can be consistently implemented at oneloop in the MSSM. The effect of radiative corrections as a function of $M_{\tilde{g}}$ and $A_{b}$ is substantial, up to several tens of $\mathrm{GeV}$ and must be considered when determining the supersymmetric parameters.

\section{Renormalization Group}

\subsection{Universal Scalar Masses at $M_{X}$}

We next consider the embedding of the MSSM in a more unified theory at a high scale, $M_{X}=\mathcal{O}\left(10^{16} \mathrm{GeV}\right)$. Having extracted the $\overline{D R}$ quantity $M_{\tilde{b}_{R}}$ from the Figs 1 , 2, 3 we would thus like to see if these values are compatible with the renormalization group running of the MSSM up to $M_{X}$. In order to qualitatively understand the evolution we first discuss an approximate analytic solution for the $M_{\tilde{b}_{R}}^{2}$ running mass. We present the full numerical analysis below. The renormalization group evolution 
of the $M_{\tilde{b}_{R}}^{2}$ mass is given [26] by,

$$
16 \pi^{2} \frac{d M_{\tilde{b}_{R}}^{2}}{d t}=4 Y_{b}^{2} \Sigma_{b}^{2}-\frac{32}{3} g_{3}^{2} M_{3}^{2}-\frac{8}{15} g_{1}^{2} M_{1}^{2}+\frac{2}{5} g_{1}^{2} \operatorname{Tr}\left(Y m^{2}\right),
$$

where

$$
\begin{aligned}
\Sigma_{b}^{2} & \equiv M_{H_{d}}^{2}+M_{\tilde{b}_{L}}^{2}+M_{\tilde{b}_{R}}^{2}+A_{b}^{2} \\
\operatorname{Tr}\left(Y m^{2}\right) & \equiv M_{H_{u}}^{2}-M_{H_{d}}^{2}+\sum_{i=1}^{n_{f}}\left(M_{\tilde{Q}_{L_{i}}}^{2}-2 M_{\tilde{u}_{R_{i}}^{2}}+M_{\tilde{d}_{R_{i}}^{2}}-M_{\tilde{L}_{L_{i}}}^{2}+M_{\tilde{e}_{R_{i}}^{2}}\right) .
\end{aligned}
$$

To start, we assume that the contribution from the bottom Yukawa coupling is small, i.e. we restrict ourselves to the region $\tan \beta \lesssim 10$. We also assume all of the squark, slepton and Higgs-boson masses are the same at the GUT scale, i.e. $\operatorname{Tr}\left(Y m^{2}\right)$ remains zero at all scales. In the case where the gluino is heavy, $M_{\tilde{g}}=200 \mathrm{GeV}$, the running of $M_{\tilde{b}_{R}}$ "freezes" below $M_{\tilde{g}}$ [26] to

$$
M_{\tilde{b}_{R}}^{2}=m_{0}^{2}+C_{3}+\frac{1}{9} C_{1}-\frac{1}{3} \sin ^{2} \theta_{w} M_{Z}^{2} \cos (2 \beta)
$$

where $m_{0}$ is the common squark, slepton and Higgs-boson mass at the GUT scale and

$$
\begin{aligned}
& C_{1}(\mu)=-\frac{2}{11} M_{1}^{2}\left[1-\frac{\alpha_{1}^{2}\left(M_{X}\right)}{\alpha_{1}^{2}(\mu)}\right], \\
& C_{3}(\mu)=\frac{8}{9} M_{3}^{2}\left[1-\frac{\alpha_{3}^{2}\left(M_{X}\right)}{\alpha_{3}^{2}(\mu)}\right] .
\end{aligned}
$$

The D-term contribution, $-\frac{1}{3} \sin ^{2} \theta_{w} M_{Z}^{2} \cos (2 \beta)$ in Eq.(4.4), is positive since $\cos (2 \beta)$ is negative, and also the bino contribution $C_{1}$ is positive. The dominant term in the evolution of $M_{\tilde{b}_{R}}^{2}$ is the gluino contribution, $C_{3}$. Now, suppose that all the scalar masses at the GUT scale are set to zero, $m_{0}=0$, and neglecting all the other positive but small contributions (proportional to $\alpha_{1}^{2}$ ) we can estimate the minimum mass of $M_{\tilde{b}_{R}}$ as

$$
M_{\tilde{b}_{R}}^{2} \gtrsim \frac{8}{9} M_{3}^{2}\left(M_{3}\right)\left[1-\frac{\alpha_{3}^{2}\left(M_{X}\right)}{\alpha_{3}^{2}\left(M_{3}\right)}\right] .
$$

From the pole gluino mass, $M_{\tilde{g}}$, we extract the $\overline{D R}$ mass [20],

$$
M_{3}\left(M_{3}\right)=M_{\tilde{g}}\left[1-\frac{15 \alpha_{3}\left(M_{3}\right)}{4 \pi}\right] .
$$

For $M_{\tilde{g}}=200 \mathrm{GeV}$, we get $M_{3}\left(M_{3}\right)=174 \mathrm{GeV}$ and

$$
M_{\tilde{b}_{R}} \gtrsim 150 \mathrm{GeV} .
$$


For larger gluino masses this becomes larger. If we allow for a positive contribution from $m_{0}^{2}$ then $M_{\tilde{b}_{R}}$ becomes correspondingly larger. From Figs. 1, 20 we see that this value is incompatible with a light sbottom of $\mathcal{O}(<10 \mathrm{GeV})$ in the small $\tan \beta \lesssim 10$ region where the above solution is valid. In fact, universality of the squark and slepton masses is incompatible with all the values of $\tan \beta$. This is because $m_{0}=0$ implies a small stop mass (even smaller than the sbottom one) excluded by the current experimental data.

Let us now consider the case of a light gluino here taken to be $3 \mathrm{GeV}$. In order to obtain chargino and neutralino masses compatible with the experimental data we keep the common electroweak gaugino mass $M_{2}=M_{1}=M_{1 / 2}$ at the GUT scale greater than $120 \mathrm{GeV}$. Then for $M_{\tilde{g}}=3 \mathrm{GeV}$ and $\alpha_{s}\left(m_{b}\right)=0.22$ we get $M_{3}\left(M_{3} \simeq m_{b}\right)=2.2 \mathrm{GeV}$. Evolving this up to the Z-scale using the relation

$$
\frac{M_{3}\left(m_{b}\right)}{\alpha_{3}\left(m_{b}\right)}=\frac{M_{3}\left(M_{Z}\right)}{\alpha_{3}\left(M_{Z}\right)},
$$

we obtain $M_{3}\left(M_{Z}\right)=1.2 \mathrm{GeV}$ which in turn gives from (4.4)

$$
M_{\tilde{b}_{R}} \simeq m_{0}
$$

That is compatible with the $M_{\tilde{b}_{R}}$ mass of our Fig. 3 for positive $m_{0}$ at the GUT scale but again is not compatible with the experimental bound on the top squark mass (> $120 \mathrm{GeV}$ ). Thus we conclude here that a light sbottom mass of order $\lesssim \mathcal{O}(10 \mathrm{GeV})$ is incompatible within the MSSM under the assumption of universality of scalar masses as well as universality of the electroweak gaugino masses at the GUT scale. The gaugino mass universality is not essential.

\subsection{Non-Universal Scalar Masses at $M_{X}$}

Analytical solutions of the renormalization group equation (4.1) in the case of nonuniversal boundary conditions have been obtained in [27], under the assumption of a small bottom Yukawa coupling and thus small $\tan \beta$ values. Even in this approximation the results are quite complicated. The term $\operatorname{Tr}\left(Y m^{2}\right)$ of (4.3) is now non-zero at the GUT scale and below. One must thus consider the running of the other soft masses as well. The coupled system of differential equations is difficult to solve.

Since we are interested in solutions of the RGE's even in the large $\tan \beta$-regime we solve them numerically. Instead of solving the RGE's assuming a specific pattern for the soft breaking masses at the GUT scale, we use our results from Figures $1 \mathrm{~b}$ and 3a for the $\overline{D R}$ right handed soft bottom mass at the Z-scale and run this up to the GUT scale together with all the other masses and couplings. We use two-loop RGE's for all the couplings and masses and full treatment of threshold effects [28]. All the other parameters have been taken to satisfy the current experimental constraints.

The dominant effect on the running of $M_{\tilde{b}_{R}}^{2}$ is the gluino mass. As we run the RGE's up in scale, the gluino mass drives $M_{\tilde{b}_{R}}^{2}$ to negative values. The scale where 
$M_{\tilde{b}_{R}}^{2}$ becomes negative depends on $\tan \beta$ and on the initial $M_{\tilde{b}_{R}}^{2}$. Note that the positive bottom Yukawa coupling contribution compensates the negative ones from the gluino in (4.1) ${ }^{4}$.

$M_{\tilde{b}_{R}}^{2}<0$ implies a charge and colour breaking minimum of the scalar potential (CCB). Note that the CCB we obtain is above a given scale, i.e. at the weak scale or below charge and colour symmetry are restored. Such a CCB at high scales (as opposed to present scales [29]) is not observationally excluded to our knowledge [30], however it would substantially alter the conventional cosmology at high temperatures. If colour and charge are broken close to the electroweak scale we would expect there to be experimentally observable effects. It is beyond the scope of this letter to investigate this in detail. In the following we shall consider charge and colour breaking to be excluded for scales $Q<1 \mathrm{TeV}$.

In order to avoid CCB we must go to large initial values of $M_{\tilde{b}_{R}}^{2}$. As we saw in our approximate analytical solution, the only possibility then of getting a light bottom squark (pole) mass is to go to large values of $\tan \beta$. This is confirmed by our full numerical calculations. In the following Table we summarize the lower bound on $\tan \beta$ for a given gluino mass for which there is no CCB at all scales below $Q<1 \mathrm{TeV}$. In fact there is also an upper bound on $\tan \beta$. For large values of $\tan \beta$ the light sbottom squared pole mass becomes negative. In other words the gluino contribution to the physical sbottom mass is such that there is always a tachyon. The upper bound on $\tan \beta$ is also summarized in the Table below.

\begin{tabular}{|c|c|c|c|c|}
\hline$M_{\tilde{g}}$ & $200 \mathrm{GeV}$ & $250 \mathrm{GeV}$ & $300 \mathrm{GeV}$ & $350 \mathrm{GeV}$ \\
\hline $\tan \beta>$ & 19 & 23 & 28 & 33 \\
\hline $\tan \beta<$ & 53 & 48 & 40 & 34 \\
\hline
\end{tabular}

So for $M_{\tilde{g}}=200 \mathrm{GeV}$ we only get a light bottom squark without CCB for $\tan \beta>19$ and we avoid a tachyonic sbottom for $\tan \beta<53$. For $M_{\tilde{g}}>350 \mathrm{GeV}$ there is no physical light sbottom mass in the MSSM. One could rather expect this result : A light sbottom of order of $10 \mathrm{GeV}$ or lighter is potentially unstable under the radiative corrections of particles with mass of a few hundred $\mathrm{GeV}$.

Next, we consider the case where we require that there is no CCB for all values $Q<M_{X}$, i.e. up to the GUT scale. The corresponding excluded parameter range is shown as the large shaded region in Figs. 1b and 3a for a heavy and light gluino, respectively. For a heavy gluino, we see that in order to retain the conventional cosmology we must require $\tan \beta>32$ and $M_{\tilde{b}_{R}}>90 \mathrm{GeV}$. For a heavier gluino, $M_{\tilde{g}}>300 \mathrm{GeV}$, the entire $\tan \beta<50$ plane is excluded. For a light gluino (Fig 3a) this excluded region is substantially reduced. Also $M_{\tilde{b}_{R}}^{2}(Q)$ turns negative only very close to the GUT scale at about $Q \sim 5 \times 10^{15} \mathrm{GeV}$ thus possibly avoiding most cosmological constraints.

\footnotetext{
${ }^{4}$ Non-universality affects also the results through the term $\operatorname{Tr}\left(Y m^{2}\right)$ in (4.1)
} 
In summary, in the case of a heavy gluino in order to have a light bottom squark we typically obtain CCB. The heavier the gluino the lower the scale at which CCB is obtained. CCB below $1 \mathrm{TeV}$ is only avoided for large values of $\tan \beta$, as summarized in (4.11). In order to avoid CCB altogether we must go to very high values of $\tan \beta$ and $M_{\tilde{b}_{R}}$ as shown in Fig. 1b. These constraints are largely avoided for a light gluino, as seen in Fig. 3a.

So far we have not considered radiative electroweak symmetry breaking (RESB) [31]. This is potentially a very strict requirement. As we saw, for a heavy gluino we required large $\tan \beta$ in order to obtain a light bottom squark. This leads to a large bottom quark Yukawa coupling such that possibly both $M_{H_{u}}^{2}$ and $M_{H_{d}}^{2}$ are negative. This is inconsistent with electroweak symmetry breaking. A systematic check of this constraint is beyond the scope of this letter. However, we would expect it to possibly be important.

\section{Light Gluino}

As we have discussed, the introduction of a light gluino naturally allows for a light $M_{\tilde{b}_{R}}$ even for small values of $\tan \beta$, while avoiding any CCBs. However, it appears that such a light gluino is experimentally excluded. In order to discuss this we distinguish between a decaying and a non-decaying light gluino. The latter could for example be the LSP. We first discuss the case of a decaying light gluino.

Until fairly recently, there was a window in the search for a decaying light gluino after combining several sets of experimental data [32, 33]. However, this window has now been closed [34] by new data from KTeV [35] and from LEP [36, 24]. We do not consider it any further.

Next we consider a stable light gluino. (It would be stable if it were the LSP.) It would have been produced in the early universe and would have a non-vanishing relic density today. These relic gluinos could bind with nuclei (possibly after forming a bound state, such as $R^{0} \equiv \tilde{g} g$ ) leading to anomalously heavy nuclei. The number of such nuclei depends on the relic density (which is a function of the self-annihilation cross section) and also on the binding potential with nuclei (which depends on the scattering cross section with nuclei). The relic density was first considered in [37]. The resulting number of anomalously heavy nuclei present today were shown to be excluded by existing searches in [38]. More recently this problem has been revisited with more detailed work on the binding potential with nuclei [39], however with the same conclusion, excluding a stable gluino 40 .

In 41] the self-annihilation cross section of the gluinos was reinvestigated. The authors concluded that unknown non-perturbative effects could possibly lead to a larger cross section and thus a significantly smaller relic density. This could possibly

avoid the bounds from anomalous heavy nuclei searches. The authors take this as a motivation to re-examine bounds from colliders. Using existing analyses from LEP 
(OPAL [42]) and the Tevatron (CDF [43]) they exclude the full range of gluino masses from $3 \mathrm{GeV}-130 \mathrm{GeV} .^{5}$

\section{Conclusions}

We have investigated the case of whether a light bottom squark $\mathcal{O}(<10) \mathrm{GeV}$ can be accommodated in the unconstrained MSSM. In our analysis we have included all the relevant one-loop corrections to the physical sbottom pole mass. For $\tan \beta \lesssim 15$ these corrections are large and need to be included when determining the physical parameters. The main effect is from the heavy gluino mass, but also the trilinear coupling $A_{b}$ leads to significant effects. In this precise framework, we were able to extract the running parameters and evolve them up to higher scales with the full two-loop RGEs including all threshold effects. In detail we find:

- If we assume universal scalar masses at the GUT scale (minimal supergravity scenario) we find a light sbottom is inconsistent with the experimental bounds on the other supersymmetric scalars. Thus in this scenario a light sbottom is excluded.

- A light sbottom can be embedded in the MSSM with non-universal scalar boundary conditions at the GUT scale only for specific conditions. For a heavy gluino $\left(M_{\tilde{g}}>180 \mathrm{GeV}\right)$ it requires large values of of $\tan \beta>30$, in order to avoid CCB at scales below $Q<1 \mathrm{TeV}$. This lower bound on $\tan \beta$ grows with the gluino mass, (4.11). For each gluino mass there is also an upper bound on $\tan \beta$ beyond which the light sbottom mass becomes tachyonic, (4.11). Above $M_{\tilde{g}}>350 \mathrm{GeV}$ a light sbottom is completely excluded. The gluino mass is thus restricted to the range $180 \mathrm{GeV}<M_{\tilde{g}}<350 \mathrm{GeV}$.

- If we require the absence of CCBs up to the GUT scale the allowed values of $M_{\tilde{g}}$ and $\tan \beta$ are significantly more restricted as summarized in Fig. 1b. Gluino masses above $300 \mathrm{GeV}$ are already excluded.

- A light sbottom could be embedded naturally in the MSSM with a light gluino $\sim 3 \mathrm{GeV}$ in a less fine tuned way avoiding also CCB constraints for almost all the $\tan \beta$ values. However, a light gluino seems to be experimentally unlikely.

\footnotetext{
${ }^{5}$ There is a possible window between $25 \mathrm{GeV}-35 \mathrm{GeV}$ for an "unlikely" set of parameters 41, which is not of direct interest to our problem. The phenomenological consequences of this window have been further explored in 44.
} 
We conclude that a light sbottom hypothesis is not completely excluded in the MSSM but it is disfavoured.

Note Added: After completing this paper, hep-ph/0008321 by Plehn and Nierste was put on the net. It is complementary to our work focusing on effects in the B-meson data for specific bottom decays to sbottoms.

\section{Acknowledgments}

We would like to thank G. Weiglein and Sven Heinemeyer for discussions on his related paper and R.G. Roberts for discussions on the running of $\alpha_{s}$. We thank John Ellis, Glennys Farrar, R. Mohapatra and Keith Olive for discussions of the light gluino. We also thank the RAL experimentalists Bill Murray, Rob Edgecock and Alberto Ribbon for discussions on the recent experimental LEP and CDF/D0 searches. We both thank the Rutherford Laboratory for an excellent working atmosphere and a great time. This work was completed despite poor treatment by PPARC.

\section{References}

[1] OPAL Collaboration, talk given at ICHEP2000, Osaka, July 2000, OPAL Physics Note 435.

[2] M. Espirito Santo, et al., DELPHI Collaboration, talk given at ICHEP2000, Osaka, July 2000, DELPHI 2000-087, CONF 386.

[3] L3 Collaboration, talk given at ICHEP2000, Osaka, July 2000, L3 Note 2601.

[4] G. Ganis, ALEPH Collaboration, talk given at ICHEP2000, Osaka, July 2000, ALEPH 2000-065 CONF 2000-043.

[5] Debajyoti Choudhury, H. Dreiner, P. Richardson, S. Sarkar, Phys. Rev. D61 (2000) 095009, hep-ph/9911365.

[6] John Ellis, K.A. Olive, S. Sarkar, D.W. Sciama, Phys. Lett. B215 (1988) 404; M. Kachelriess, JHEP 0002 (2000) 010, hep-ph/0001160.

[7] J. Ellis, S. Rudaz, Phys. Lett. B128 (1983) 248; K. Hikasa, M.Kobayashi, Phys. Rev. D36 (1987) 724; M. Drees, K. Hikasa, Phys. Lett. B252 (1990) 127; G. Mahlon, G.L. Kane, Phys. Rev. D55 (1997) 2779, hep-ph/9609210; CDF Collaboration (T. Affolder et al.) Phys. Rev. Lett. 84 (2000) 5273, hep-ex/9912018; D0 Collaboration (S. Abachi et al.), Phys. Rev. Lett. 76 (1996) 2222; M. Antonelli, G. Sguazzoni, ALEPH Collaboration, talk given at ICHEP2000, Osaka, July 2000, CERN-EP/2000-085.

[8] D0 Collaboration (B. Abbott et al.), Phys. Rev. D60 (1999) 031101, hep-ex/9903041. 
[9] CDF Collaboration (T. Affolder et al.), Phys. Rev. Lett. 84 (2000) 5704, hepex/9910049.

[10] G. Abbiendi et al. , OPAL Collaboration, Phys. Lett. B456 (1999) 95, hepex/9903070.

[11] J. Abdallah et al. , DELPHI Collaboration, contribution at ICHEP2000, Osaka, July 2000, DELPHI 2000-090, CONF 389.

[12] W. Da Silva et al. , DELPHI Collaboration, contribution at ICHEP2000, Osaka, July 2000, DELPHI 2000-096, CONF 395.

[13] L3 Collaboration, contribution at ICHEP2000, Osaka, July 2000, L3 Note 2587.

[14] See in particular Fig. 7 in [12].

[15] ALEPH Collaboration, the last reference in 7 .

[16] S. Pacetti, Y. Srivastava, hep-ph/0007318.

[17] M. Carena, S. Heinemeyer, C.E.M. Wagner, G. Weiglein, hep-ph/0008023.

[18] Particle Data group, D.E. Groom et al, Eur. Phys. J. C 15 (2000) 1.

[19] A. Dedes, S. Moretti, Nucl. Phys. B 576 (2000) 29; hep-ph/9909418.

[20] D. M. Pierce, J. A. Bagger, K. Matchev, R.-J. Zhang, Nucl. Phys. B 491 (1997) 3, hep-ph/9606211.

[21] "A Supersymmetry Primer", Stephen P. Martin, published in "Perspectives on supersymmetry", World Scientific, edited by G. Kane, hep-ph/9709356.

[22] LEP Electroweak Working Group, http://lepewwg.web.cern.ch/LEPEWWG/stanmod/lepew99.ps.gz

[23] J. Ellis, D.V. Nanopoulos, D.A. Ross, Phys. Lett. B305 (1993) 375, hep-ph/9303273; R.G. Roberts and J. Stirling, Phys. Lett. B313 (1993) 453, hep-ph/9306244; M. Jezabek, J. H. Kuhn, Phys. Lett. B301 (1993) 121, hep-ph/9211322; M. Schmelling, R.D. St. Denis, Phys. Lett. B329 (1994) 393.

[24] F. Csikor, Z. Fodor, Phys. Rev. Lett. 78 (1997) 4335, hep-ph/9611320.

[25] D0 Collaboration (B. Abbott et al.), Phys. Rev. Lett 83 (1999) 4937, hep-ex/9901023.

[26] S. P. Martin and P. Ramond, Phys. Rev. D48 (1993) 5365 hep-ph/9306314.

[27] P. Nath and R. Arnowitt, Phys. Rev. D56 (1997) 2820 hep-ph/9701301.

[28] A. Dedes, A. B. Lahanas and K. Tamvakis, Phys. Rev. D53 (1996) 3793, hepph/9504239. 
[29] See for example S.A. Abel, C.A. Savoy, Phys. Lett. B444 (1998) 119, hep-ph/9809498; S. Abel, T. Falk, Phys. Lett. B444 (1998) 427, hep-ph/9810297 and references therein.

[30] For an overview on bounds see for example Subir Sarkar, Rept. Prog. Phys. 59 (1996) 1493, hep-ph/9602260.

[31] L. Ibanez, G. G. Ross, Phys. Lett. B110 (1982) 215;

K. Inoue, A. Kakuto, H. Komatsu and S. Takeshita, Progr. Theor. Phys. 68 (1982) 927, 71 (1984) 96;

L. Alvarez-Gaumé, M. Claudson and M. Wise, Nucl. Phys. B207 (1982) 96;

J. Ellis, D.V. Nanopoulos and K. Tamvakis, Phys. Lett. B121 (1983) 123.

[32] See the summary by H. Murayama, in the Particle Data Group Review, 1998, C. Caso, et al. , Eur. Phys. J. C3 (1998) 1.

[33] For a summary of the light gluino bounds in 1994 see G. R. Farrar, hep-ph/9408379.

[34] G.R. Farrar, talk at SUSY 97, Philadelphia, PA, 27-31 May 1997, Nucl. Phys. Proc. Suppl. 62 (1998) 485, hep-ph/9710277; private communication Aug. 2000.

[35] KTeV Collaboration, A. Alavi-Harati et al., Phys. Rev. Lett. 83 (1999) 2128, hepex/9903048; KTeV Collaboration, J. Adams et al., Phys. Rev. Lett. 79 (1997) 4083, hep-ex/9709028.

[36] Z. Nagy, Z. Trocsanyi, Phys. Rev. D57 (1998) 5793, hep-ph/9712385; hep-ph/9708343; P. Abreu et al (Delphi Collaboration), Phys. lett. B414 (1997) 401; R. Barate et al (Aleph Collaboration), Z. Phys. C96 (1997) 1.

[37] C.B. Dover, T.K. Gaisser, G. Steigman, Phys. Rev. Lett. 42 (1979) 1117; S. Wolfram, Phys. lett. B82 (1979) 65.

[38] J. Ellis, J.S. Hagelin, D.V. Nanopoulos, K. Olive, M. Srednicki, Nucl. Phys. B238 (1984) 453;

[39] R.N. Mohapatra, V.L. Teplitz, Phys. Rev. Lett. 81 (1998) 3079, hep-ph/9804420; R.N. Mohapatra, F. Olness, R. Stroynowski, V.L. Teplitz, Phys. Rev. D60 (1999) 115013, hep-ph/9906421

[40] R. Mohapatra, privat communication.

[41] H. Baer, K. Cheung, J.F. Gunion, Phys. Rev. D59 (1999) 075002, hep-ph/9806361.

[42] OPAL Collaboration (G. Alexander et al.), Phys. Lett. B377 (1996) 273.

[43] CDF Collaboration (F. Abe et al.), Phys. Rev. D56 (1997) 1357.

[44] S. Raby, K. Tobe, Nucl. Phys. B539 (1999) 3, hep-ph/9807281; A. Mafi, S. Raby, Phys. Rev. D62 (2000) 035003, hep-ph/9912436. 